The Journal of $\mathbf{N}_{\text {onlinear }} \mathbf{S}_{\text {cience and }}$ A pplications

http://www.tjnsa.com

\title{
A NEW REGULARITY CRITERION FOR THE NAVIER-STOKES EQUATIONS
}

\author{
HU YUE ${ }^{1 *}$ AND WU-MING LI ${ }^{2}$ \\ This paper is dedicated to Themistocles M. Rassias on the occasion of his sixtieth birthday \\ Communicated by Choonkil Park
}

Abstract. We study the incompressible Navier-Stokes equations in the entire three-dimensional space. We prove that if $\partial_{3} u_{3} \in L_{t}^{s_{1}} L_{x}^{r_{1}}$ and $u_{1}, u_{2} \in L_{t}^{s_{2}} L_{x}^{r_{2}}$, then the solution is regular. Here $\frac{2}{s_{1}}+\frac{3}{r_{1}} \leq 1,3 \leq r_{1} \leq \infty, \frac{2}{s_{2}}+\frac{3}{r_{2}} \leq 1$ and $3 \leq r_{2} \leq \infty$.

\section{INTRODUCTION AND PRELIMINARIES}

In this paper, we consider Cauchy problem for the incompressible Navier-Stokes equations

$$
\left\{\begin{aligned}
\frac{\partial u}{\partial t}+u \cdot \nabla u+\nabla p & =\nu \triangle u \\
\nabla \cdot u & =0 \\
u(x, 0) & =u_{0}(x)
\end{aligned}\right.
$$

in which $u=\left(u_{1}(x, t), u_{2}(x, t), u_{3}(x, t)\right)$ is the unknown velocity field, $u_{0}=$ $\left(u_{1}(x, 0), u_{2}(x, 0), u_{3}(x, 0)\right)$ is the initial velocity field with $\nabla \cdot u_{0}=0$, and $p(x, t)$ is a scalar pressure. While $\nu$ is the kinematic viscosity coefficient, we will assume that $\nu \equiv 1$ for simplicity in this paper. Here we use the classical notations

$$
\nabla u=\left(\partial_{1} u, \partial_{2} u, \partial_{3} u\right), \triangle u=\sum_{i=1}^{3} \partial_{i}^{2} u, \nabla \cdot u=\sum_{i=1}^{3} \partial_{i} u_{i}, u \cdot \nabla u=\sum_{j=1}^{3} u_{j} \partial_{j} u
$$

Date: Received: August 12, 2010; Revised: November 24, 2010.

*Corresponding author

(C) 2011 N.A.G.

2000 Mathematics Subject Classification. Primary 35Q30, 76D03.

Key words and phrases. Navier-Stokes equations; Leray-Hopf weak solution; Regularity. 
In 1934, Leray proved the existence of a global weak solution

$$
u \in L^{\infty}\left(0, \infty ; L^{2}\left(\mathbb{R}^{3}\right)\right) \bigcap L^{2}\left(0, \infty ; H^{1}\left(\mathbb{R}^{3}\right)\right),
$$

on the condition that $u_{0} \in L^{2}$ and $\nabla \cdot u_{0}=0$. It is called the Leray-Hopf weak solution which satisfies the energy inequality [1]. If $\|u(t)\|_{H^{1}}$ is continuous, then we say that $u(t)$ is regular. Up to the present, the regularity and uniqueness of the weak solutions is still an open problem. On the other hand, there are already many criterions which ensure that the weak solution is regular $[2,3]$.

In this paper, we give a regularity criterion concerning one derivative of one component of the velocity and other components of velocity.

\section{MAin Results}

Our main result can be stated as follows.

Theorem 2.1. Let

$$
\partial_{3} u_{3} \in L_{t}^{s_{1}} L_{x}^{r_{1}}, \text { where } \frac{2}{s_{1}}+\frac{3}{r_{1}} \leq 1,3 \leq r_{1} \leq \infty,
$$

and

$$
u_{1}, u_{2} \in L_{t}^{s_{2}} L_{x}^{r_{2}}, \text { where } \frac{2}{s_{2}}+\frac{3}{r_{2}} \leq 1,3 \leq r_{2} \leq \infty .
$$

Then $u$ is regular.

At first, let us recall the definition of the Leray-Hopf weak solution.

Definition 2.2. (Leray-Hopf weak solution) If a measurable vector u satisfies the following properties in $0 \leq T \leq \infty$ :

(1) $u$ is weakly continuous in $[0, T) \times L^{2}\left(\mathbb{R}^{3}\right)$,

(2) $u$ verifies (1.1) in the sense of distribution

$$
\int_{0}^{T} \int_{\mathbb{R}^{3}}\left(\frac{\partial \Phi}{\partial t}+u \cdot \nabla \Phi+\triangle \Phi\right) \cdot u d x d t+\int_{\mathbb{R}^{3}} u_{0} \cdot \Phi(x, 0) d x=0,
$$

$\forall \Phi \in C_{0}^{\infty}\left(\mathbb{R}^{3} \times[0, T)\right), \nabla \cdot \Phi=0, \Phi$ is a vector function,

(3) $u$ satisfies the energy inequality

$$
\|u(\cdot, t)\|_{L^{2}}^{2}+2 \int_{0}^{t}\|\nabla u(\cdot, s)\|_{L^{2}}^{2} d s \leq\left\|u_{0}\right\|_{L^{2}}^{2}, 0 \leq t \leq T,
$$

then $\mathrm{u}$ is called a Leray-Hopf weak solution of the Navier-Stokes equations.

We give three lemmas for the proof of the theorem.

Lemma 2.3. (The estimate for the pressure in $L^{q}\left(\mathbb{R}^{3}\right)$ )

$$
\|p\|_{L^{q}}(t) \leq C\|u\|_{L^{2 q}}^{2}(t), 1<q<\infty .
$$

Proof. See [2]. 
Lemma 2.4. (Interpolation inequality) Assume $u \in L_{t}^{\infty} L_{x}^{2}\left(\mathbb{R}^{3} \times I\right)$ and $\nabla u \in$ $L_{t}^{2} L_{x}^{2}\left(\mathbb{R}^{3} \times I\right)$, where $I$ is an open interval. Then $u \in L_{t}^{s} L_{x}^{r}\left(\mathbb{R}^{3} \times I\right)$ for all $r, s$ such that

and $2 \leq r \leq 6$. Moreover,

$$
\frac{2}{s}+\frac{3}{r} \leq \frac{3}{2}
$$

$$
\|u\|_{L_{t}^{s} L_{x}^{r}} \leq C\|u\|_{L_{t}^{\infty} L_{x}^{2}}^{(6-r) / 2 r}\|\nabla u\|_{L_{t}^{2} L_{x}^{2}}^{(3 r-6) / 2 r}
$$

Proof. See [3].

By Lemmas 2.3 and 2.4, we get the following.

Lemma 2.5. (The estimate for the pressure in $L_{t}^{s} L_{x}^{r}\left(\mathbb{R}^{3} \times I\right)$ )

$$
\|p\|_{L_{t}^{s} L_{x}^{r}} \leq C\left\|u_{0}\right\|_{L^{2}}^{2}, \frac{2}{s}+\frac{3}{r}=3,1<r \leq 3 .
$$

Proof. (Proof of Theorem 2.1) At first, let us multiply the equation for $u_{3}$ of (1.1) by $\left|u_{3}\right| u_{3}$ and integrate over $[0, T] \times \mathbb{R}^{3} t \in(0, T)$

$$
\begin{aligned}
\int_{0}^{t} \int_{\mathbb{R}^{3}} \frac{\partial u_{3}}{\partial t} \cdot\left|u_{3}\right| u_{3}-\int_{0}^{t} \int_{\mathbb{R}^{3}} \triangle u_{3} \cdot\left|u_{3}\right| u_{3} & =-\int_{0}^{t} \int_{\mathbb{R}^{3}} u \cdot \nabla u_{3} \cdot\left|u_{3}\right| u_{3} \\
& -\int_{0}^{t} \int_{\mathbb{R}^{3}} \partial_{3} p \cdot\left|u_{3}\right| u_{3} \\
I_{1}+I_{2} & =I_{3}+I_{4} .
\end{aligned}
$$

Calculating every term,

$$
\begin{aligned}
& I_{1}=\frac{1}{3} \int_{0}^{t} \int_{\mathbb{R}^{3}} \frac{\partial\left|u_{3}\right|^{3}}{\partial t}=\left.\frac{1}{3}\left\|u_{3}\right\|_{L^{3}}^{3}\right|_{0} ^{t}=\frac{1}{3}\|u(\cdot, t)\|_{L^{3}}^{3}-\frac{1}{3}\|u(\cdot, 0)\|_{L^{3}}^{3}, \\
& I_{2}=-\sum_{i=1}^{3} \int_{0}^{t} \int_{\mathbb{R}^{3}} \partial_{i i} u_{3}\left|u_{3}\right| u_{3}=\frac{8}{9} \sum_{i=1}^{3} \int_{0}^{t} \int_{\mathbb{R}^{3}}\left(\partial_{i}\left|u_{3}\right|^{3 / 2}\right)^{2}=\frac{8}{9}\left\|\nabla\left|u_{3}\right|^{3 / 2}\right\|_{L_{t}^{2} L_{x}^{2}}^{2}, \\
& I_{3}=-\sum_{i=1}^{3} \int_{0}^{t} \int_{\mathbb{R}^{3}} u_{i} \partial_{i} u_{3}\left|u_{3}\right| u_{3}=\frac{1}{3} \sum_{i=1}^{3} \int_{0}^{t} \int_{\mathbb{R}^{3}} \partial_{i} u_{i}\left|u_{3}\right|^{3}=0, \\
& I_{4}=\int_{0}^{t} \int_{\mathbb{R}^{3}} p \partial_{3}\left(\left|u_{3}\right| u_{3}\right) \leq C\|p\|_{L_{t}^{s_{2}} L_{x}^{r_{2}}}^{3 / 2}\left\|\partial_{3} u_{3}\right\|_{L_{t}^{s_{1}} L_{x}^{r_{1}}}^{3 / 2}+\frac{1}{6}\left\|u_{3}\right\|_{L_{t}^{\infty} L_{x}^{3}}^{3},
\end{aligned}
$$

where

$$
\left\{\begin{array}{lll}
\frac{1}{s_{1}}+\frac{1}{s_{2}}=1 & \\
\frac{1}{r_{1}}+\frac{1}{r_{2}}=\frac{2}{3} & \\
\frac{2}{s_{1}}+\frac{3}{r_{1}}=1 & 2 \leq s_{1} \leq \infty, 3 \leq r_{1} \leq \infty \\
\frac{2}{s_{2}}+\frac{3}{r_{2}}=3 & 1 \leq s_{2} \leq 2, \frac{3}{2} \leq r_{2} \leq 3
\end{array}\right.
$$

By Lemma 2.3, we get

$$
\|p\|_{L_{t}^{s_{2}} L_{x}^{r_{2}}} \leq C\left\|u_{0}\right\|_{L^{2}}^{2}
$$

and so

$$
I_{4} \leq C\left\|u_{0}\right\|_{L^{2}}^{3}\left\|\partial_{3} u_{3}\right\|_{L_{t}^{s_{1}} L_{x}^{r_{1}}}^{3 / 2}+\frac{1}{6}\left\|u_{3}\right\|_{L_{t}^{\infty} L_{x}^{3}}^{3} .
$$


Summarizing the above calculating of $I_{1}, I_{2}, I_{3}$ and $I_{4}$, we get

$$
\frac{1}{6}\left\|u_{3}(\cdot, t)\right\|_{L^{3}}^{3}+\frac{8}{9}\left\|\nabla\left|u_{3}\right|^{\frac{3}{2}}\right\|_{L_{t}^{2} L_{x}^{2}}^{2} \leq C\left\|u_{0}\right\|_{L^{2}}^{3}\left\|\partial_{3} u_{3}\right\|_{L_{t}^{s_{1}} L_{x}^{r_{1}}}^{\frac{3}{2}}+C\left\|u_{0}\right\|_{L^{2}}^{\frac{3}{2}}\left\|\nabla u_{0}\right\|_{L^{2}}^{\frac{3}{2}} .
$$

By (2.1), we get

$$
\left\|u_{3}(\cdot, t)\right\|_{L_{t}^{\infty} L_{x}^{3}}+\left\|\nabla\left|u_{3}\right|^{\frac{3}{2}}\right\|_{L_{t}^{2} L_{x}^{2}} \leq C
$$

moreover,

and so

$$
\left\|u_{3}(\cdot, t)\right\|_{L_{t}^{3} L_{x}^{9}} \leq C\left\|\nabla\left|u_{3}\right|^{\frac{3}{2}}\right\|_{L_{t}^{2} L_{x}^{2}}^{\frac{2}{3}}
$$

$$
\left\|u_{3}(\cdot, t)\right\|_{L_{t}^{\infty} L_{x}^{3}}+\left\|u_{3}(\cdot, t)\right\|_{L_{t}^{3} L_{x}^{9}} \leq C,
$$

i.e., $u_{3} \in L_{t}^{s} L_{x}^{r}$, where $\frac{2}{s}+\frac{3}{r} \leq 1,3 \leq r \leq 9$, so the solution is regular making use of the regularity criterion $[4,5]$ :

$$
u \in L_{t}^{s} L_{x}^{r}, \text { where } \frac{2}{s}+\frac{3}{r} \leq 1,3 \leq r \leq \infty,
$$

which completes the proof.

Acknowledgements: This work was supported by NSFC Grant 10771052.

\section{REFERENCES}

1. J. Leray, Sur le mouvement d'un liquide visqueux emplissant l'espace, Acta Math. 63 (1934), 193-248. 1

2. D. Chae and J. Lee, Regularity criterion in terms of pressure for the Navier-Stokes equations, Nonlinear Anal.-TMA 46 (2001), 727-735. 1, 2

3. I. Kukavica and M. Ziane, Navier-Stokes equations with regularity in one direction, J. Math. Phys. 48 (2007), Art. ID 065203. 1, 2

4. J. Serrin, On the interior regularity of weak solutions of the Navier-Stokes equations, Arch. Ration. Mech. Anal. 9 (1962), 187-195. 2

5. L. Escauriaza, G. Seregin and V. S̆verk, On backward uniqueness for parabolic equations, Zap. Nauch. Seminarov POMI 288 (2002), 100-103. 2

1 School of Mathematics and Information Science, Henan Polytechnic UniverSity, Jiaozuo, Henan 454000, China.

E-mail address: huu3y2@163.com

2 School of Mathematics and Information Science, Henan Polytechnic UniverSity, JiaOzuo, HenAN 454000, China.

E-mail address: liwum0626@126.com 